\title{
STUDY ON CHARACTERIZATION OF Ni/BIOCHAR CATALYST DERIVED FROM MICROALGAL BIOMASS
}

\author{
Vuong Van Pham ${ }^{1}$, Hong Khanh Dieu Nguyen ${ }^{2, *}$ \\ ${ }^{I}$ Vietnam Oil and Gas Group, No. 18 Lang Ha street, Hanoi city \\ ${ }^{2}$ Hanoi University of Science and Technology, No. 1 Dai Co Viet street, Hanoi city \\ "Email: dieuhong_bk@yahoo.com
}

Received: 16 January 2016; Accepted for publication: 2 August 2016

\begin{abstract}
Biochar supported nickel (Ni/biochar) catalyst was prepared by incipient wetness method and characterized by using a series of techniques such as XRD, SEM, TEM, FT-IR, $\mathrm{H}_{2}$-TPR and BET. These charaterizations indicated the catalyst structure and demonstrated its potential for applications in reduction - oxidation reactions in particular the HDO process.
\end{abstract}

Keywords: carbon supported nickel, HDO, microalgal biomass, biochar.

\section{INTRODUCTION}

Nowaday most of catalysts applied for hydrodeoxygenation (HDO) process belonged to those used for the hydrodesufurization (HDS) process because of their correspondences in the reaction mechanisms. These catalysts contained various reduction metals such as $\mathrm{Co}$, Mo or $\mathrm{Ni}$ supported on aluminium oxides, and the metals could be partially sulfurized for stabiliazing their activity and avoiding the catalysts poisons [1]. The generation of catalysts still showed good activity for the HDO processes, but the expenses for preparations, stabilizations and activations considerably restricted their applications. On the other hand a series of different catalysts were also invented such as NiW, noble metal based catalysts and $\mathrm{FeS}$, but the major disadvantages still be concerned to their complicate preparation processes and high cost [2 - 4].

Pyrolyzing of biomass could produce two kinds of main products including biochar and bio-oil; in which, the bio-oil as feedstock played an important role in the HDO processes, but the biochar has not been used effectively yet. In fact the biochar could be important for soil nutrition improvement at present days, but it could be also modified by different techniques for preparing catalysts or adsorbants in the chemical technology. The structure of biochar belonged to the intermediate between that of carbohydrates in the biomass and graphite with systems of condensed polycyclic aromatic rings [5]. This property provided the biochar the ability for easily modifying because of the high surface tension of the rings. This led to our idea to impregnating some active metals such as $\mathrm{Ni}, \mathrm{Co} \ldots$ on the biochar surface; then the as-synthesized materials could be applied in the HDO process of the mentioned bio-oil. The Ni portions generated after hydrogen reduction of the synthesized catalyst played a crucial role in the activity of the $\mathrm{Ni} /$ biochar catalyst because there were the high active metal sites located on the biochar support. 
The idea approached a close loop process using the microalgal biomass as feedstock and producing the hydrocarbons as the main product.

In a previous paper [6], the biochar supporting $\mathrm{Ni}$ catalyst ( $\mathrm{Ni} /$ biochar) was prepared through incipient wetness method. The catalysts activity was also tested in the HDO process with the feedstock bio-oil showing good performance when reducing a large amount of the beginning oxygen content from the feedstock to the products. In this paper, the catalysts characterizations were investigated for clarifying the correspondence between the catalysts structure and properties and its ability on oxygen removal in the HDO process.

\section{EXPERIMENTALS}

\subsection{Chemicals and equipments}

$\mathrm{Ni}\left(\mathrm{NO}_{3}\right)_{2}$ was purchased from Merck for directly using without any further purification. Biochar was obtained through thermal pyrolysis of microalgal biomass type Botryococcus. $\mathrm{H}_{2} / \mathrm{N}_{2}$ supplier was purchased from Shanghai Eternal Faith Industry Co., Ltd., China.

\subsection{Preparation of $\mathrm{Ni} / \mathrm{biochar}$ catalyst}

The catalyst Ni/biochar was prepared in a previous paper [6] following the two-step procedure including pyrolysis of microalgal biomass, wetness impregnation of $\mathrm{Ni}\left(\mathrm{NO}_{3}\right)_{2}$ at mass percentage of $20 \%$ onto biochar, calcination at $400{ }^{\circ} \mathrm{C}$ for 4 hours and reduction $\mathrm{Ni}^{2+}$ to $\mathrm{Ni}^{\circ}$ using $\mathrm{H}_{2} / \mathrm{N}_{2}$ gas mixture at $400{ }^{\circ} \mathrm{C}$ for 3 hours. The impregnation was finished by vaporizing the rest of water for maintaining the $\mathrm{Ni}$ content in the final catalyst.

The Ni/biochar catalyst was applied in the HDO process using feedstock bio-oil obtained from the pyrolysis in other studies. In this paper the Ni/biochar catalysts were established by many techniques such as XRD, SEM, TEM, FT-IR, $\mathrm{NH}_{3}$-TPD and BET.

\subsection{Techniques for characterizing the $\mathrm{Ni} / \mathrm{biochar}$ catalyst}

Many techniques including XRD, SEM, TEM, FT-IR, $\mathrm{NH}_{3}$-TPD, $\mathrm{H}_{2}$-TPR and BET were used in the study for characterizing the biochar and Ni/biochar catalyst. The XRD was recorded in D8 Advance - Bruker; the SEM and TEM images were collected using Field Emission Scaning Electron Microscope S - 4800 and JEOL 1100 respectively; the FT-IR spectroscopy was recorded using Nicolet 6700 FT-IR Spectrometer; the $\mathrm{NH}_{3}$-TPD and $\mathrm{H}_{2}$-TPR were measured in AutoChem II 2920 Micromeritics and Micromeritics AutoChem II 2920 V4.01 respectively and the BET method was established using Chem BET -3000 .

\section{RESULTS AND DISCUSSION}

\subsection{XRD patterns}

Figure 1 described the XRD patterns of the biochar and Ni/biochar catalyst. Observations exhibited that both biochar and catalyst existed in amorphous phases with high background intensity. The patterns also poined out none of any crystalline phases although the $\mathrm{Ni}\left(\mathrm{NO}_{3}\right)_{2}$ content in the beginning mass ratio was up to $20 \% \mathrm{wt}$. There were two ways to explain this phenomenon: the first reason was that $\mathrm{NiO}$ and portions generated in the catalysts calcination 
and reduction homogeneously distributed on the biochar surface with extreme thin layers not being detected by XRD detector; the second reason was that the $\mathrm{NiO}$ and $\mathrm{Ni}$ portions possessed the amorphous phases, or in the other hand, that could be said that the Ni portions on the surface existed in a metallic glass state [7].

The latter was more reasonable because BET surface area of the biochar was not high enough for extremely homogeneous distributions (referred to the results obtained from BET technique in 3.6), especially with very high content of the provided Ni precursor. Otherwise, there was a considerable difference in the background shape of the XRD pattern between the biochar and the catalyst caused by the different nature of amorphous phase of the Ni portions and the biochar. Incase of the mentioned homogeneous distribution, they should be the same.
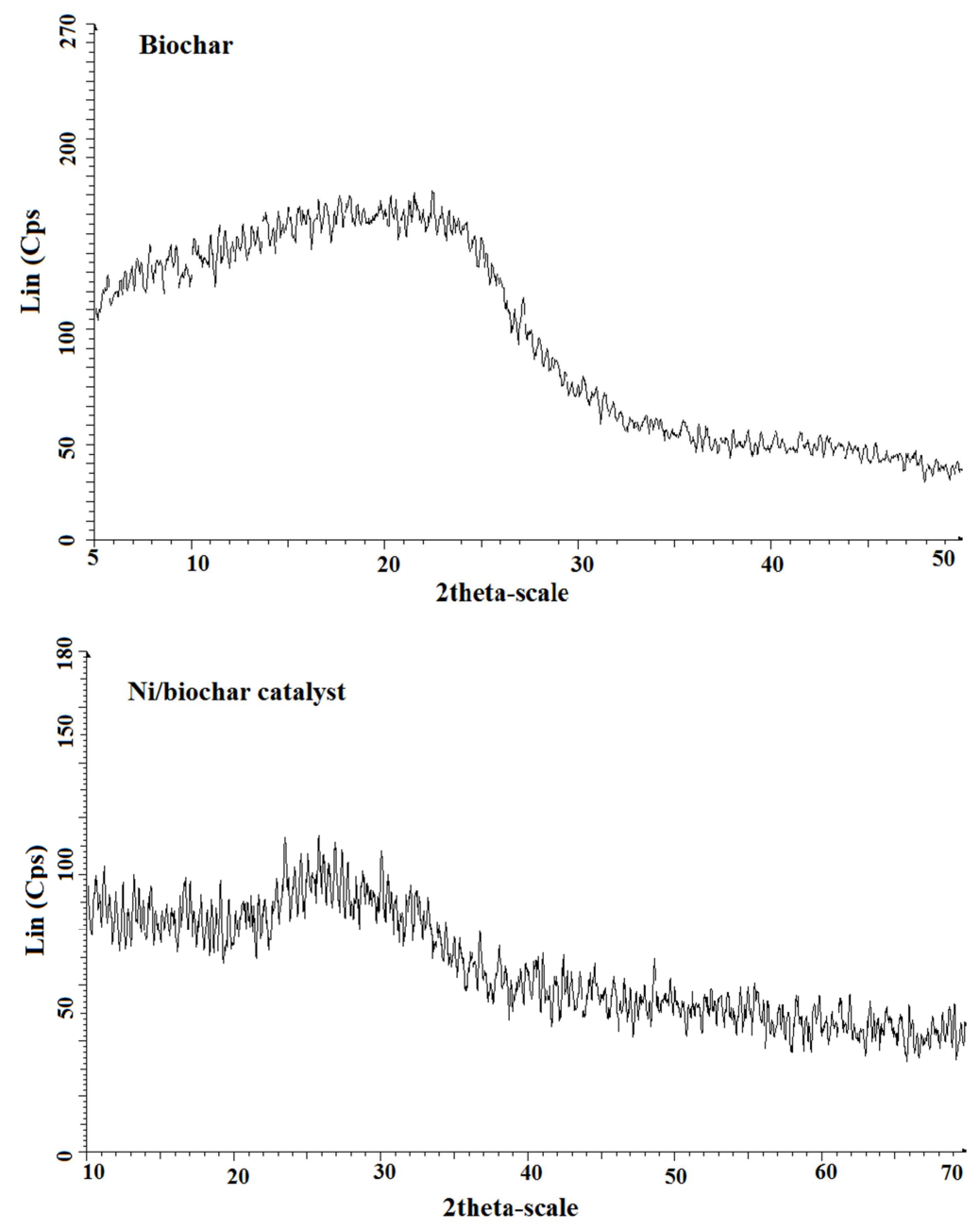

Figure 1. XRD patterns of biochar and catalyst Ni/biochar derived from microalgal biomass.

The decomposition reaction of the $\mathrm{Ni}\left(\mathrm{NO}_{3}\right)_{2}$ and the reduction of the $\mathrm{NiO}$ portions were illustrated as followed reaction equations:

$$
\begin{gathered}
2 \mathrm{Ni}\left(\mathrm{NO}_{3}\right)_{2}=2 \mathrm{NiO}+4 \mathrm{NO}_{2}+\mathrm{O}_{2} \\
\mathrm{NiO}+\mathrm{H}_{2}=\mathrm{Ni}+\mathrm{H}_{2} \mathrm{O}
\end{gathered}
$$




\subsection{SEM images}
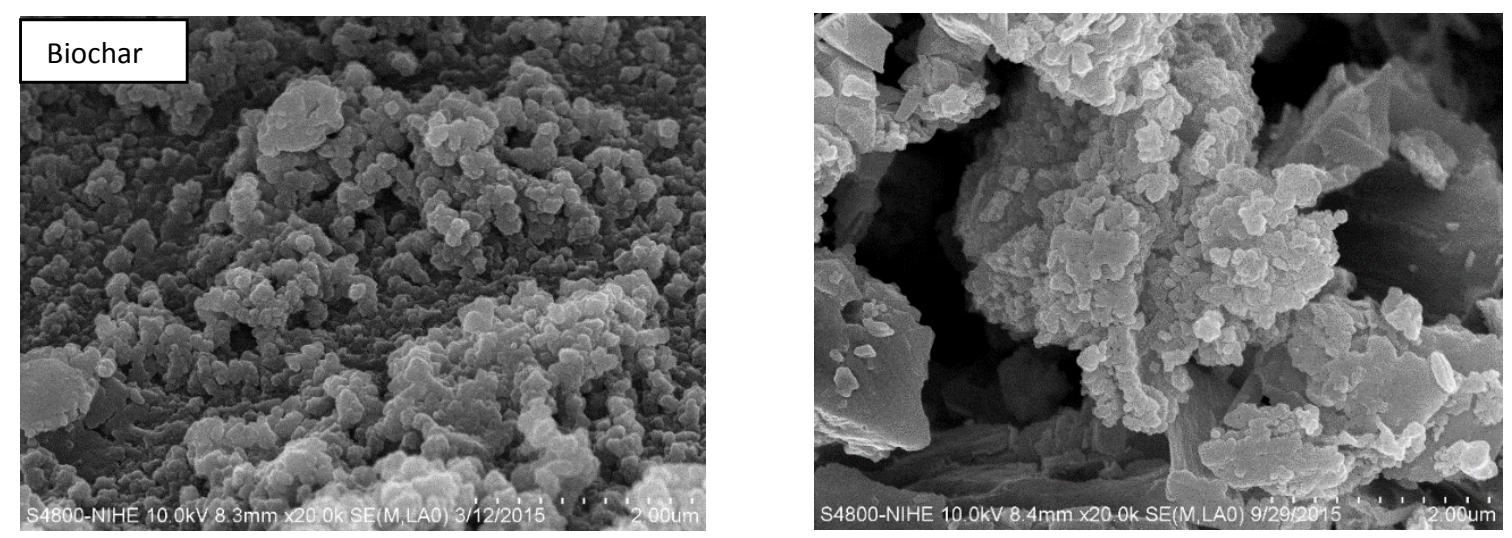

Figure 2. SEM images of the biochar and the Ni/biochar catalyst.

Figure 2 showed the SEM images of the biochar and the Ni/biochar catalyst. In the same implications, the SEM images clearly indicated difference in morphology of the two materials: the biochar mainly contained uniform $20 \mathrm{~nm}$ sized particles agglomerated together producing a large clusters while the $\mathrm{Ni} /$ biochar catalyst consisted of small thin size particles arranged in the layer structure beside the same clusters as the biochars morphology. The observations were well adaptive to the different background shapes of their XRD patterns confirming that the $\mathrm{NiO}$ and Ni portions on the catalyst surface existed in the amorphous states. The Ni distribution was well looked in the TEM images.

\subsection{TEM images of the Ni/biochar catalyst}

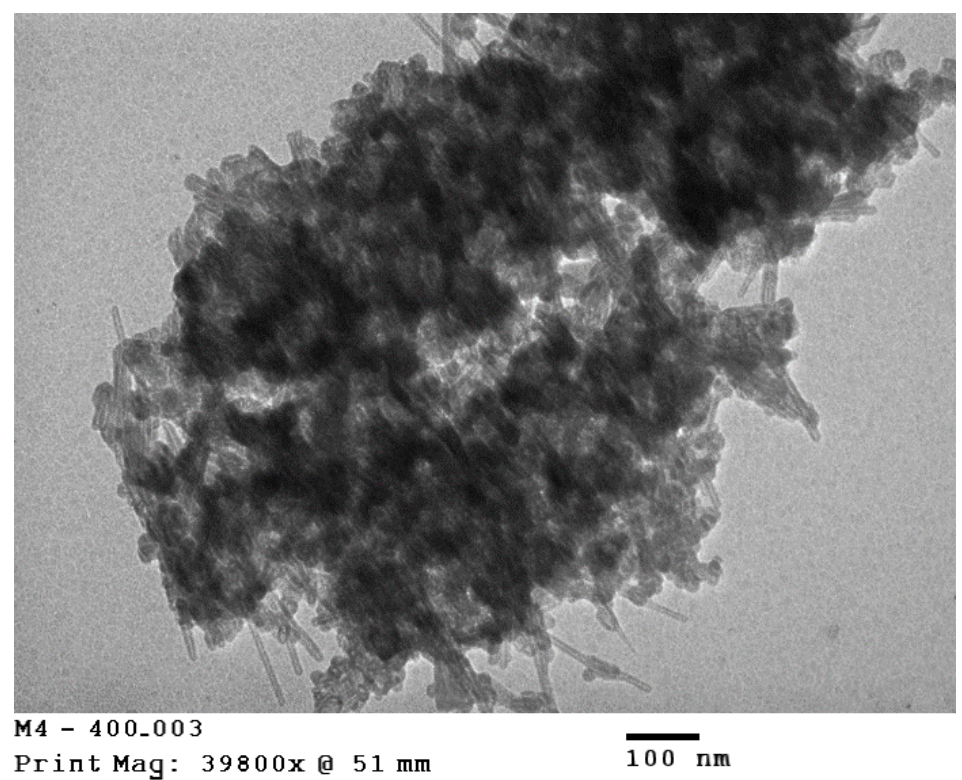

Figure 3. TEM image of the Ni/biochar catalyst. 
Figure 3 showed the TEM image of the Ni/biochar catalyst. There was many dark regions distributed along with light background corresponded to the Ni clusters on the biochar surface respectively. The dark regions with size about $30-50 \mathrm{~nm}$ could be assigned for the agglomeration of the $\mathrm{Ni}$ metals during the calcination and the reduction of the Ni/biochar catalyst. An interesting observation was obtained when occurring some long bar morphologies indicating the formation of carbon nanotubes $[7,8]$. This structure could be generated during the pyrolysis of the microalgal biomass. The carbon nanotubes possessed mesoposous structure, so they could be useful for increasing diffusion ability of the Ni clusters on the catalysts surface and avoiding the overagglomeration of the active sites during the calcination.

\subsection{FT-IR spectroscopies}

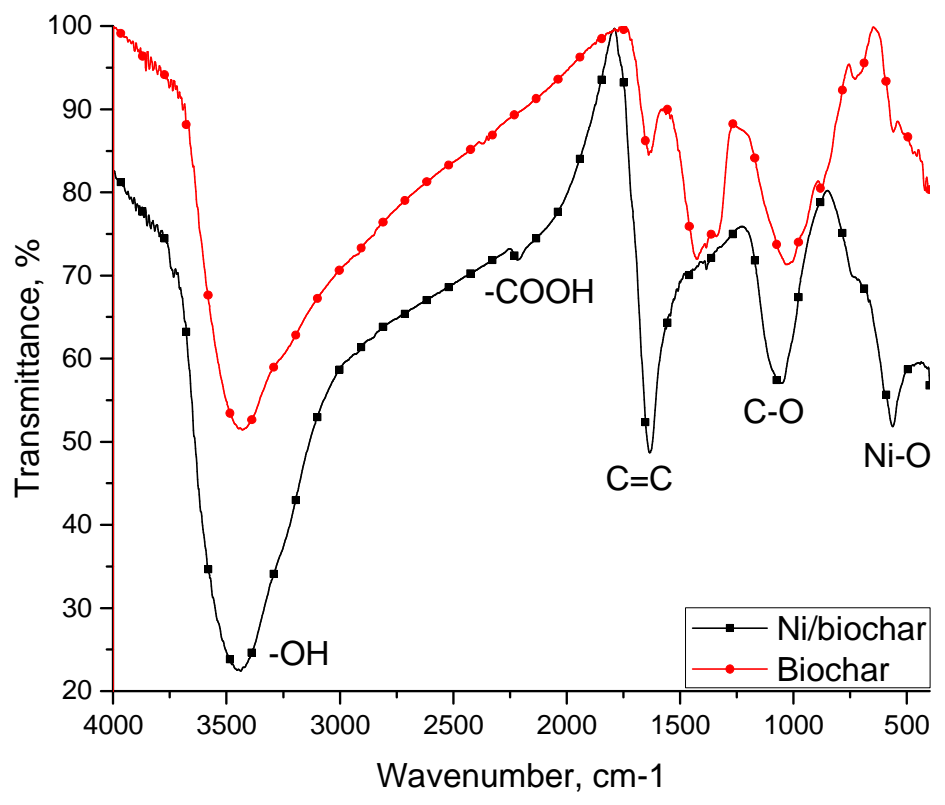

Figure 4. FT-IR spectroscopies of the biochar and Ni/biochar catalyst.

Figure 4 revealed the FT-IR spectroscopy of the biochar and Ni/biochar catalyst. The analysis results pointed out apprearences of many the same organic groups in the polycyclic aromatic system of the biochar and catalyst including $-\mathrm{OH}$ phenolic groups at $\sim 3400 \mathrm{~cm}^{-1},-\mathrm{OH}$ carboxylic acid at $\sim 2200 \mathrm{~cm}^{-1}$ and $\mathrm{C}=\mathrm{C}$ aromatic rings at $\sim 1650 \mathrm{~cm}^{-1}$. These results reflected the same framework of the biochar and the catalyst. Especially with the Ni/biochar catalyst, there were additions of Ni-O vibration at $\sim 510 \mathrm{~cm}^{-1}$ assigning for a redshift from that of the bulk $\mathrm{NiO}$ at $\sim 460 \mathrm{~cm}^{-1}$ [9] demonstrating the apprearence of $\mathrm{Ni}-\mathrm{O}-\mathrm{C}$ bonds formed by $\mathrm{NiO}$ and the support through oxygen brigdes. The partial transference of electron density from Ni-O to the polycyclic aromatic system coud be corresponded for this phenomenon. There was also an absence of Ni-C connections illustrating none of the carbon brigdes occurring like $\mathrm{Ni}-\mathrm{C}_{\text {support }}$ connections. In addition, the redshift could not be observed when considering the $\mathrm{Ni}-\mathrm{C}_{\text {support }}$ because the $\mathrm{Ni}^{2+}$ portions always attracted the electron from the aromatic system through the conjugation effect reducing the electron density of this system.

\section{5. $\mathrm{H}_{2}$-TPR ananlysis}




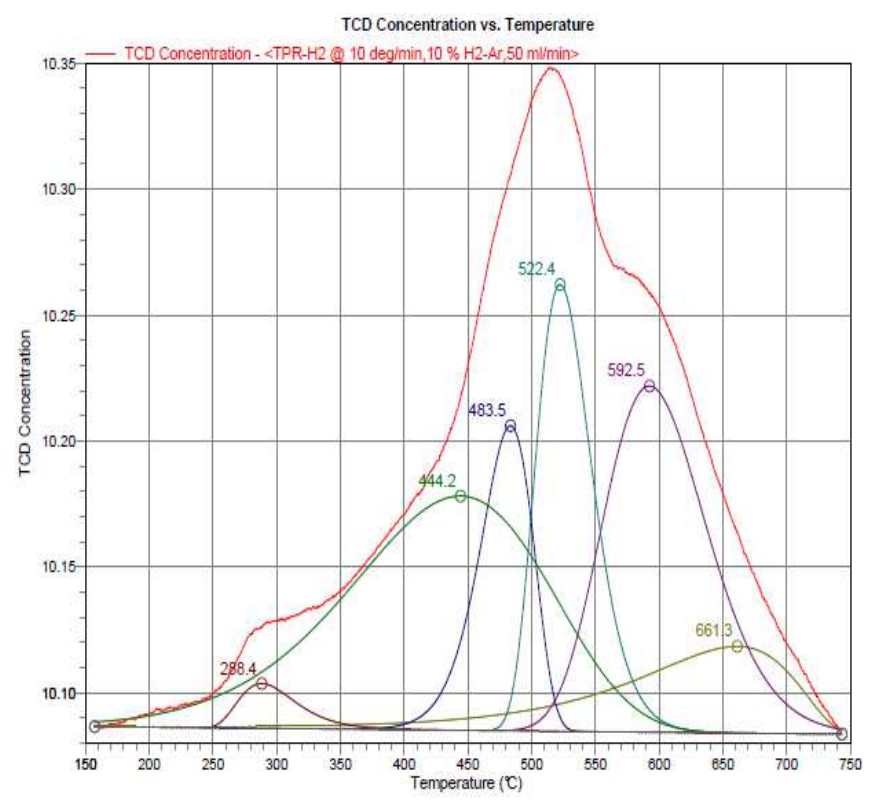

\begin{tabular}{|c|c|}
\hline Reduction peaks ${ }^{\circ} \mathrm{C}$ & $\mathrm{H}_{2}$ consumptions, mmol/g \\
\hline 288.4 & 0.06943 \\
\hline 444.2 & 1.28235 \\
\hline 483.5 & 0.45003 \\
\hline 522,4 & 0.68493 \\
\hline 592.5 & 0.97467 \\
\hline 661.3 & 0.40975 \\
\hline
\end{tabular}

Figure 5. Diagram and results from $\mathrm{H}_{2}-$ TPR of the Ni/biochar.

$\mathrm{NiO}$ as a product of the decomposition of $\mathrm{Ni}\left(\mathrm{NO}_{3}\right)_{2}$ could be existed on the biochar surface either bulk or connected with support. Normally, the bulk NiO tended to agglomerate during the calcination because of their weak connection to the support producing the very large size clusters on the surface and lowering the activity of the catalyst in the oxidation-reduction reactions, particulally the HDO reaction. Contrarily, if the connections between the biochar and the $\mathrm{NiO}$ portions became too strong, the reduction of the $\mathrm{NiO}$ could be hard to establish and required high temperature for a long time - a critical factor for increasing the agglomeration of the active sites. Therefore, a suitable connection with intermediate bond energy between the biochar and the $\mathrm{NiO}$ was highly recommended; in which the reduction temperature ranged from 400 to $\sim 500{ }^{\circ} \mathrm{C}$. The $\mathrm{H}_{2}$-TPR technique was applied to estimate this reduction ability of the catalyst, and this was also related to the activity of the generated Ni portions in the HDO process. The $\mathrm{H}_{2}$-TPR diagram and results were decribed in Figure 5.

The results showed 6 reduction peaks corresponding to 6 states of the connections between the $\mathrm{NiO}$ and the biochar at different temperatures. In which, the peak at $288.4{ }^{\circ} \mathrm{C}$ could be assigned to the reduction of the bulk $\mathrm{NiO}$ portions weakly attaching to the support; the peak at $444.2{ }^{\circ} \mathrm{C}, 483.5{ }^{\circ} \mathrm{C}$ and $522.4{ }^{\circ} \mathrm{C}$ corresponded to the $\mathrm{NiO}$ portions connected with the biochar at medium strength through Ni-O-C $\mathrm{C}_{\text {support }}$ (referred to the FT-IR spectroscopy analysis in 3.4); the peak at $592.5^{\circ} \mathrm{C}$ and $661.3^{\circ} \mathrm{C}$ characterized for the $\mathrm{NiO}$ portions with strong bonds with the support. As given mention above, the connections with medium strength between the $\mathrm{NiO}$ with biochar were the most suitable for the Ni/biochar catalysts activity. Assuming that all the $\mathrm{NiO}$ portions were reduced from $\mathrm{Ni}^{2+}$ to $\mathrm{Ni}^{\mathrm{o}}$, then the generated $\mathrm{Ni}$ site density was equal to the moles of the consumed hydrogen during the reduction. Therefore, the amount of the suitable connected $\mathrm{NiO}$ portions with medium strength took an account for $(1.28235+0.45003+$ $0.68493) /(0.06943+1.28235+0.45003+0.68493+0.97467+0.40975)=62.44 \%$ of the total amount of the $\mathrm{NiO}$ located on the biochar surface. The high percentage of the active $\mathrm{NiO}$ portions on the biochars surface considerably enhanced the catalysts activity in the oxidationreduction processes.

\subsection{BET measurements}




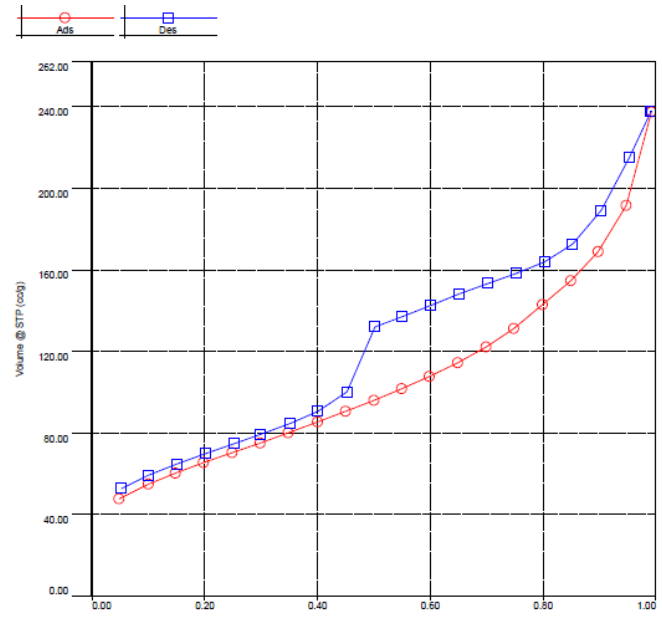

Figure 6. Adsorption - desorption isotherms of the Ni/biochar catalyst.

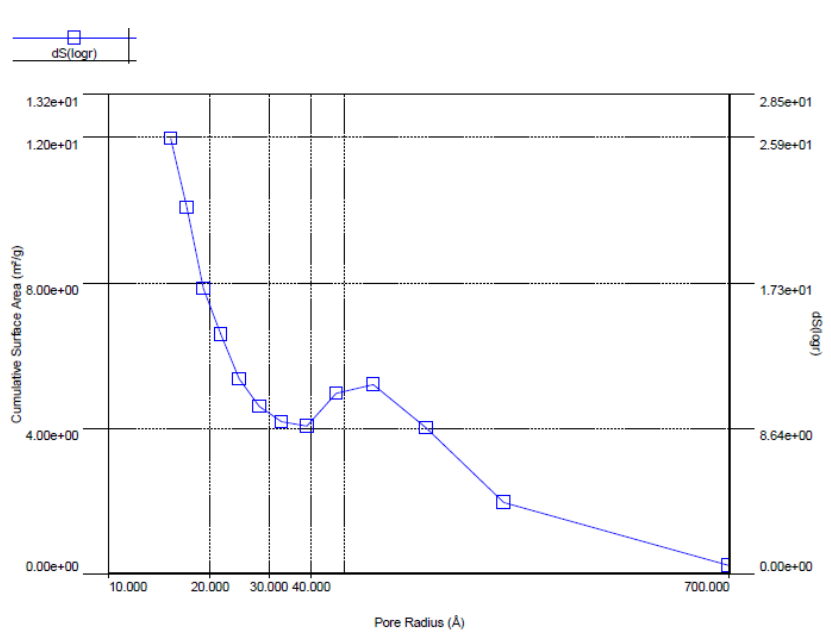

Hình 7. Pores distribution of the Ni/biochar catalyst.

The adsorption - desorption isortherm of the Ni/biochar catalyst indicated a large hysteresis between the adsorption and desorption isotherms including the characterization of the appreared mesoporous structure. The BET surface area reached $65.712 \mathrm{~m}^{2} / \mathrm{g}$ proved that the amount of the mesopores were not as high as some typical mesoporous materials such as MCM-41 or SBA-15. The pore distribution also confirmed these conclusions because it showed a range of pore diameters focusing at $40 \AA$. The mesopores could be assigned for the generation of the carbon nanotubes observed in the TEM image of the catalyst. Otherwise, the pore distribution haven't concentrated on the narrow diameters demonstrating the amount of the carbon nanotubes was low.

\section{CONCLUSION}

The Ni/biochar catalyst possessed amorphous phases generated through combination of biochar, $\mathrm{NiO}$ and Ni clusters. The catalyst also contained a dominative amount of mediumstrength interactions between the $\mathrm{NiO}$ clusters and the biochar through $\mathrm{Ni}-\mathrm{O}-\mathrm{C}$ connections. The texture and morphology properties of the catalyst showed the existence of mesoporous structure and a small supprised amount of coexisted carbon nanotubes. The high content of the connected $\mathrm{NiO}$ portions located on the biochar surface could play an important role in strengthening the activity of the catalyst in the HDO process.

\section{REFERENCES}

1. Bulusheva D. A., Rossa J. R. H. - Catalysis for conversion of biomass to fuels via pyrolysis and gasification: A review, Catalysis Today 171 (2011) 1- 13.

2. Elliott D. C. - Historical Developments in Hydroprocessing Bio-oils, Energy Fuels 21 (2007) 1792-1815. 
3. Wildschut J., Mahfud F. H.,Venderbosch R. H., Heeres H. J. - Hydrotreatment of Fast Pyrolysis Oil Using Heterogeneous Noble-Metal Catalysts, Ind. Eng. Chem. Res. 48 (2009) 10324-10334.

4. Rocha J. D., Luengo C. A., Snape C. E. - The scope for generating bio-oils with relatively low oxygen contents via hydropyrolysis, Org. Geochem. 30 (1999) 1527-1534.

5. Rosalind E. Franklin - Crystallite Growth in Graphitizing and Non-Graphitizing Carbons, Proc. R. Soc. Lond. A 209 (1951) 196-218.

6. Vuong Van Pham, Hong Khanh Dieu Nguyen - Preparation of Ni/Biochar obtained from microalgal biomass for hydrodeoxygenation of pyrolysis oil, Proceedings the $2^{\text {nd }}$ International conference on Chemmical engineering, Food and Biotechnology ICCFB2015 (2015) 123-130.

7. Xian-fa Lia, Xue-gang Luo - Preparation of Mesoporous Activated Carbon Supported Ni Catalyst for Deoxygenation of Stearic Acid into Hydrocarbons, Environmental Progress \& Sustainable Energy 34 (2) (2015) 607-612.

8. Göksel Özkan, Serdar Gök, Gülay Özkan - Active carbon-supported $\mathrm{Ni}, \mathrm{Ni} / \mathrm{Cu}$ and $\mathrm{Ni} / \mathrm{Cu} / \mathrm{Pd}$ catalyzed steam reforming of ethanol for the production of hydrogen, Chemical Engineering Journal 171 (2011) 1270- 1275.

9. Assem Barakat, Mousa Al-Noaimi, Mohammed Suleiman, Abdullah S. Aldwayyan, Belkheir Hammouti, Taibi Ben Hadda, Salim F. Haddad, Ahmed Boshaala, Ismail Warad - One Step Synthesis of NiO Nanoparticles via Solid-State Thermal Decomposition at Low-Temperature 